\title{
Medición de la calidad en el servicio: una perspectiva desde la Educación Superior Pública
}

\section{Measuring quality in service: a perspective from Public Higher Education}

\author{
TORRES-RIVERA, Ma. Patricia †*, TRISTAN-MONRROY, Beatriz Virginia y FLORES-RUEDA, \\ Isabel Cristina
}

Universidad Autónoma de San Luis Potosí, Coordinación Académica Región Altiplano, México.

ID $1^{\text {er }}$ Autor: Ma. Patricia, Torres-Rivera / ORC ID: 0000-0001-6122-476X, CVU CONACYT ID: 524977

ID $1^{\mathrm{er}}$ Coautor: Beatriz Virginia, Tristan-Monrroy / ORC ID: 0000-0002-9022-8118; CVU CONACYT ID: 969512

ID $2^{\mathrm{do}}$ Coautor: Isabel Cristina, Flores-Rueda / ORC ID: 0000-0003-1257-2893, CVU CONACYT ID: 1001753

DOI: $10.35429 / J U P .2020 .11 .4 .9 .15$

Recibido: 15 de Enero, 2020; Aceptado 30 de Junio, 2020

\begin{abstract}
Resumen
La presente investigación de enfoque cuantitativo, no experimental, de corte transversal y descriptivo se realizó a una Institución de Educación Superior (IES) de la Región Altiplano en el estado de San Luis Potosí, cuyo objetivo es medir la calidad en el servicio acorde a las percepciones y expectativas de las áreas transversales mediante el modelo Servqual. La población objetivo de estudio fueron los 1,287 estudiantes inscritos en los siete programas educativos de la Coordinación Académica Región Altiplano (COARA) de la UASLP y los 129 académicos y administrativos que laboran en la institución. Los resultados muestran como la evaluación por parte de los usuarios varia en función de sus necesidades y expectativas. Se identificó que la dimensión con una mejor evaluación es la de Capacidad de Respuesta, seguida de la Empatía, Seguridad, Fiabilidad y Elementos Tangibles, indicándonos así el orden de importancia y las áreas de mejora dentro de la institución. Por último, se da una evaluación general a la institución con una media de 4.18 puntos. Los alumnos tienen una percepción mas favorable respecto a la calidad en el servicio comparado con los académicos y administrativos.
\end{abstract}

Calidad en el servicio, Servqual, Institución Educativa Superior

\begin{abstract}
The present research with a quantitative, nonexperimental, cross-sectional and descriptive approach was carried out at an Institution of Higher Education (IES) of the Altiplano Region in the state of San Luis Potosí, whose objective is to measure the quality of the service according to the Perceptions and expectations of the transversal areas through the Servqual model. The target population of the study was the 1,287 students enrolled in the seven educational programs of the Highland Region Academic Coordination (COARA) of the UASLP and the 129 academic and administrative workers who work at the Institution. The results show how the evaluation by users vary according to their needs and expectations. It was identified that the dimension with the best evaluation is Responsiveness, followed by Empathy, Security, Reliability and Tangible Elements, thus indicating the order of importance and areas for improvement within the institution. Finally, a general evaluation is given to the institution with an average of 4.18 points. The students have a more favorable perception regarding the quality of the service compared to the academic and administrative.
\end{abstract}

Service quality, Servqual, Higher Educational Institution

Citación: TORRES-RIVERA, Ma. Patricia, TRISTAN-MONRROY, Beatriz Virginia y FLORES-RUEDA, Isabel Cristina. Medición de la calidad en el servicio: una perspectiva desde la Educación Superior Pública. Revista de Políticas Universitarias. 2020. 4-11:9-15.

\footnotetext{
* Correspondencia al Autor (Correo electrónico: patricia.torres@uaslp.mx)

$\dagger$ Investigador contribuyendo como primer autor.
} 


\section{Introducción}

La globalización ha desarrollado cambios significativos tanto en los sistemas económicos, políticos y sociales, así como en el sector público de la eduación. Por lo anterior, las Instituciones de Educación Superior (IES) en México se ven en la necesidad de responder a demandas exigentes que presenta el contexto nacional e internacional; se enfrentan a retos de cobertura, pertinencia y calidad que requieren modelos administrativos y académicos flexibles que les permita tener una capacidad de respuesta confiable y eficaz. Una educación de calidad contribuye a disminuir la brecha de desigualdad y favorece las oportunidades entre la comunidad.

La economía de México se caracteriza por la diversidad regional y su creciente apertura. Se prevé que los sectores estratégicos del país (aeroespacial, del automóvil, de la energía y de la electrónica) mantengan sus trayectorias de crecimiento, a lo que hay que sumar las reformas en curso para incrementar la productividad y la innovación también en sectores más tradicionales. La educación superior está creciendo y, si se mantienen los patrones actuales, el $26 \%$ de los jóvenes obtendrán algún título de educación superior a lo largo de su vida. Medio millón de egresados ingresan cada año en el mercado laboral y México confía en ellos para progresar en las cadenas de valor mundiales (OECD, 2019).

El propósito de la medición de la calidad en el servicio de las organizaciones es conocer y desarrollar estrategias a partir de las evaluaciones de los clientes y así, mantenerse, consolidarse o crecer de forma competitiva en el sector.

En el presente estudio se revisan y presentan de una manera descriptiva el concepto de calidad en el servicio desde el modelo de evaluación SERVQUAL de la escuela americana planteados por Parasuraman, Zeithaml y Berry (1993), donde se propone la medición de la calidad en el servicio percibido desde la fiabilidad, sensibilidad, seguridad, empatía y elementos tangibles.

\section{Revisión bibliográfica}

\section{Importancia de las IES}

La educación superior es el nivel con que culmina nuestro sistema educativo, lo cual implica que no debe ser considerado en forma aislada, sino en relación con los ciclos educativos que le preceden. Esta posición del sistema de educación superior exige que de su planeación se esperen resultados de organización racional y funcionamiento eficiente, adecuadamente vinculados a los de niveles que le son previos. Consecuentemente, las metas y objetivos que se propongan para mejorar las funciones de la educación superior, a nivel institucional, regional o nacional, formarán parte de la totalidad del sistema educativo nacional que, desde todo punto de vista, es un componente indivisible del desarrollo integral del país (Asociación Nacional de Universidades e Instituciones de Educación Superior [ANUIES], 2019).

\section{La Calidad en el Servicio}

Un elemento esencial para el entendimiento del concepto de calidad en el servicio es el concepto inherente servicio a cliente. Para Duque y Diosa (2014) es una relación donde está implícita la satisfacción mutua tanto del establecimiento y su desempeño en la entrega del producto como las expectativas del cliente. Ahora bien, para conocer las expectativas del servicio es necesario que las organizaciones tomen en cuenta los procesos de prestación del servicio y la retroalimentación de los empleados, clientes y proveedores. La "calidad del servicio" según Malcom Peel (2010); a quien señalamos por la claridad de su idea, puede ser descifrado como "aquella actividad que relaciona la empresa con el cliente a fin de que éste quede satisfecho con dicha actividad".

Percepción Según Schiffman y Kanuk (2005), la percepción se define como el proceso mediante el cual un individuo selecciona, organiza e interpreta los estímulos para formarse una imagen significativa y coherente del mundo. Dos individuos podrían estar expuestos a los mismos estímulos en las mismas condiciones, sin embargo, la forma en que cada uno de ellos reconoce, selecciona, organiza e interpreta constituye un proceso altamente singular, basado en las necesidades, valores y expectativas específicas de cada persona. 
Los autores Hill, Jones y Gareth (1996) mencionan que es la satisfacción del cliente visto desde el punto de vista de cumplir con el cliente en tiempo oportuno, es decir, el "logro de una capacidad de respuesta de los clientes, el tiempo que se requiere para entregar un producto o servicio (a mayor tiempo, mayor insatisfacción del cliente).

El Modelo SERVQUAL, mide la calidad del servicio, mediante las expectativas y percepciones de los clientes, con base a cinco dimensiones, que son; dimensión de fiabilidad, sensibilidad, seguridad, empatía, y elementos tangibles (Nishizawa, 2018).

SERVQUAL, es una escala de ítems múltiples que sirve para medir las percepciones del cliente con respecto a la calidad del servicio (Parasuraman, 1985). El instrumento se compone de 22 frases que miden las expectativas del cliente y 22 frases similares que miden las percepciones del cliente y la calidad del servicio se establece mediante la diferencia de las puntuaciones que el cliente asignó a las expectativas y a las percepciones (Duque y Chaparro, 2012).

Según Zeithaml, Bitner y Gremler, (2009) Los elementos tangibles es la apariencia física, instalaciones físicas, como la infraestructura, equipos, materiales, personal.

\section{Metodología}

La presente investigación es de enfoque cuantitativo, no experimental, de corte transversal y descriptivo. Se desarrolló como caso de estudio en la Coordinación Académica Región Altiplano (COARA), de la Universidad Autónoma de San Luis Potosí (UASLP). La población objeto de estudio está conformada por el 100\% (1287) de la matrícula de los siete programas educativos, cinco de ellos orientados al área de las ingenierías (Ingeniería Química, Ingeniería Mecatrónica, Ingeniería Mécanico Administrador) uno orientado al área de la salud (Licenciatura en Enfermería) y el último orientado al área de la administración (Licenciatura en Mercadotecnia), y por los 129 académicos y administrativos que laboran en la institución, la muestra obtenida para el caso del personal que labora dentro de la institución fue de 88 elementos, con un nivel de confianza del $90 \%$ y un margen de error del 5\%, el método de muestreo fue no probabilístico.
Como instrumento de medición se usó el Modelo SERVQUAL, el cual mide las expectativas y percepciones de los clientes, con base a 22 items dividido en cinco dimensiones: elementos tangibles, confiabilidad, capacidad de respuesta, seguridad y empatía, en una escala Likert indicando: 5 Muy satisfecho, 4 Satisfecho, 3 Aceptable, 2 Insatisfecho y 1 Muy insatisfecho. Este instrumento se aplicó en línea a través de la plataforma de la institución a los 1287 alumnos inscritos en los siete programas que imparte la COARA y a los 88 académicos y administrativos, durante el periodo de ingreso al nuevo ciclo escolar, enero-junio 2020. Una vez recabada la información se procesó y se analizó en el paquete estadístico SPSS21.

\section{Resultados}

El análisis de los resultados se realizó en dos partes, el primer análisis muestra la evaluación general que se tiene de la institución a través de las cinco dimensiones del modelo Servqual; por parte de los alumnos y muestra la percepción de cada una de las dimensiones que tienen los estudiantes respecto a la calidad del servicio prestado por las diez áreas transversales que conforman el soporte de apoyo a la comunidad estudiantil. El segundo análisis corresponde a la evaluación que hace el personal académico y administrativo de la Institución respecto a la calidad en el servicio.

\section{Evaluación general de la Institución}

A partir del análisis general referente a la percepción que tienen los alumnos sobre la calidad del servicio que brindan las diferentes áreas; dirección, administración, secretaría académica, secretaría general, secretaría escolar, vinculación, servicio social, tutorías, deportes, Programa Institucional de Promoción a la Salud (PIPS), Centro Integral de Aprendizaje en Matehuala (CIAM) y Departamento Universitario de Inglés (DUI) de la COARA, en lo general las 5 dimensiones se ubican calificadas en rangos desde satisfecho a muy satisfecho.

Es así como, la dimensión con una mejor evaluación es la capacidad de respuesta con una media de 4.27 puntos. 
La segunda dimensión con parámetros altos de evaluación es empatía con una media de 4.2, seguridad con una media de 4.19, fiabilidad con una media de 4.17 y elementos tangibles con una media de 4.08, indicándonos así el orden de importancia dentro de la institución (ver la Figura 1. Dimensiones del Modelo Servqual).

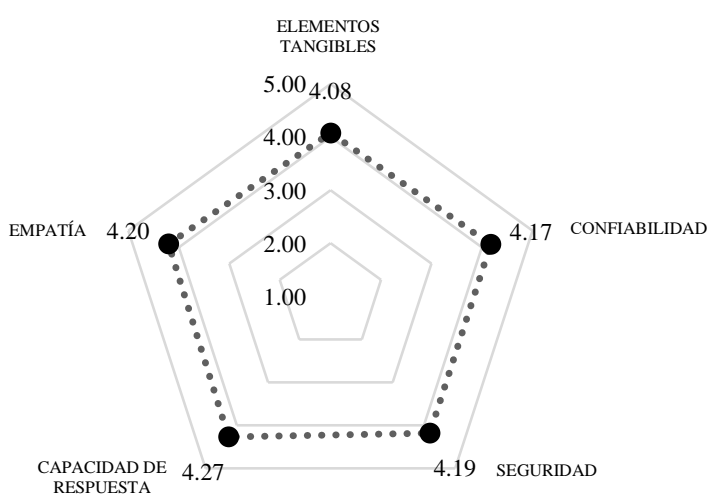

Figura 1 Dimensiones del Modelo Servqual Fuente: Elaboración propia a partir de los datos

\section{Elementos Tangibles}

Como se observa en la Figura 2 sobre elementos tangibles se lograron medir atributos como: apariencia de las instalaciones físicas, equipo, personal y materiales para comunicaciones.

Se identifica que, tanto personal como instalaciones obtuvieron una mejor evaluación, seguido del equipamiento con el cual se cuenta, mientras que la publicidad y la tecnología son los puntos de oportunidad de mejoras dentro de la institución.

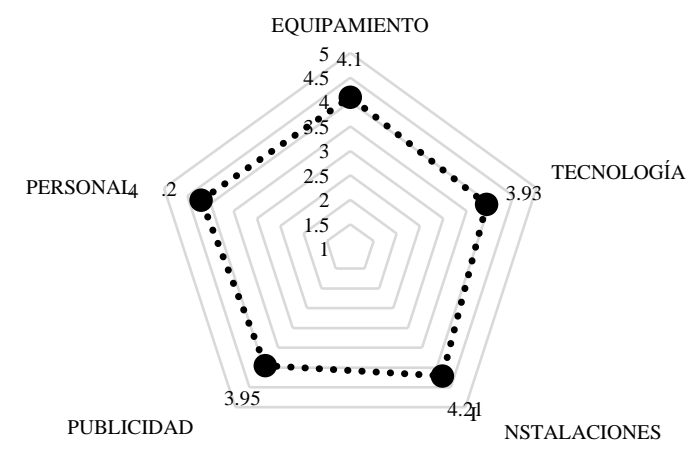

Figura 2 Dimensión Elementos Tangibles Fuente: Elaboración propia a partir de los datos.

\section{Confiabilidad}

Respecto a la directriz de confiabilidad se midió la capacidad para brindar el servicio prometido en forma precisa, digna de confianza y la manera en la que se les despejan las dudas a los estudiantes. Se observa que el área cuya evaluación sobresale es el CIAM con 4.38 puntos que se ubican en calificaciones entre muy satisfecho y satisfecho.

Consecutivamente, se revisa el DUI con una calificación de 4.26 puntos, mientras que el área de Tutorías y Servicio Social con una calificación de 4.18 puntos. Mientras que, el área de dirección es evaluada con una puntuación menor de 4.02 puntos con respecto a otras áreas evaluadas. Es importante resaltar que todas las áreas obtienen evaluaciones entre muy satisfecho y satisfecho (ver la Figura 3 Dimensión de Confiabilidad).

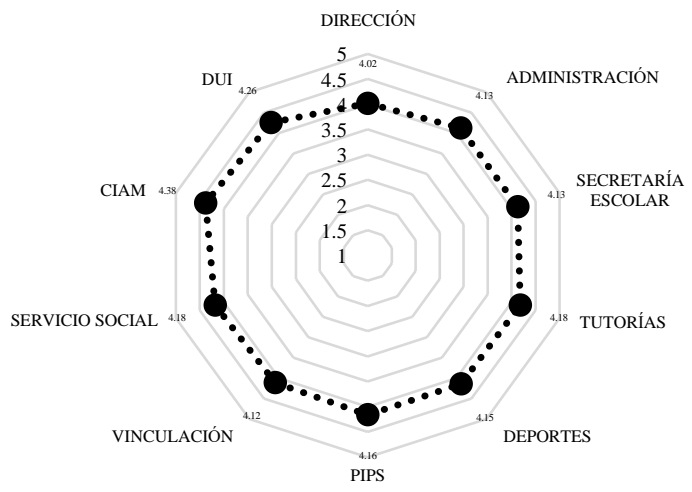

Figura 3 Dimensión de Confiabilidad

Fuente: Elaboración propia a partir de los datos.

\section{Capacidad de respuesta}

Sobre esta dimensión describe la buena disposición para ayudar a los clientes a proporcionarles un servicio expedito, es decir cuando los colaboradores del servicio tienen el deseo de ayudar a los usuarios de forma rápida y concisa. Por ello, se mide la comunicación, la rapidez y la disposición de ayudar a los alumnos. En la Figura 4 sobre capacidad de respuesta se observa que CIAM, DUI y Servicio Social son las áreas mejor evaluadas mientras que el área directiva obtiene una evaluación menor con 4.12 puntos. 


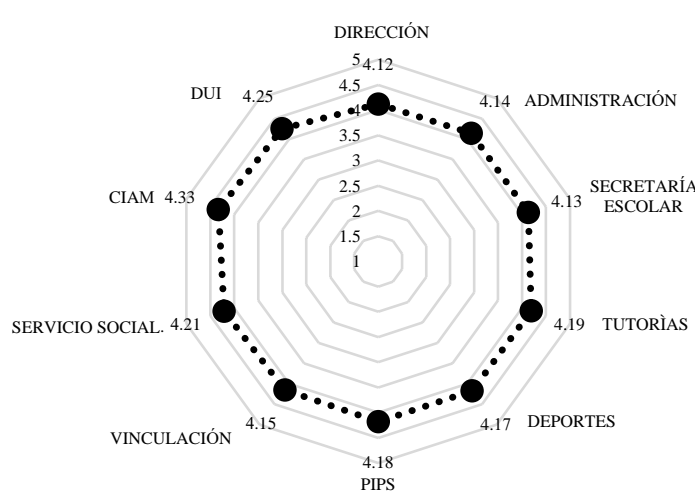

Figura 4 Capacidad de respuesta

Fuente: Elaboración propia a partir de los datos

\section{Seguridad percibida.}

En la Figura 5 se observan las 10 áreas evaluadas de la COARA en donde CIAM obtiene una calificación de 4.37, DUI se califica en promedio con 4.29 así como servicio social y secretaría escolar de la institución con una evaluación de 4.28 puntos.

Se observa que el área directiva tiene una menor calificación con respecto a las demás con 4.22 puntos. Sobresale que, las 10 áreas transversales están evaluadas con una calificación superior a la media, lo cual indica que los estudiantes se encuentran satisfechos con la directriz de seguridad percibida que ofrecen dichas áreas.

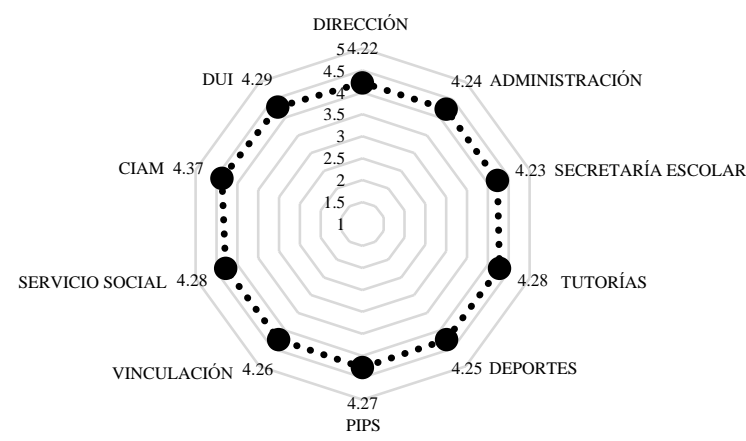

Figura 5 Dimensión de Seguridad

Fuente: Elaboración propia a partir de los datos

\section{Empatía}

Esta dimensión muestra el cuidado y atención que la institución proporciona a sus estudiantes. En la Figura 6 se observa las 10 áreas evaluadas dentro de la COARA; CIAM obtuviene una calificación superior de 4.31 puntos respecto a las áreas restantes, DUI con 4.25 puntos $\mathrm{y}$ finalmente, la gestión del servicio social con 4.22 puntos.
Complementariamente se observa que dirección y administración fueron los evaluados con menor calificación con 4.16 puntos.

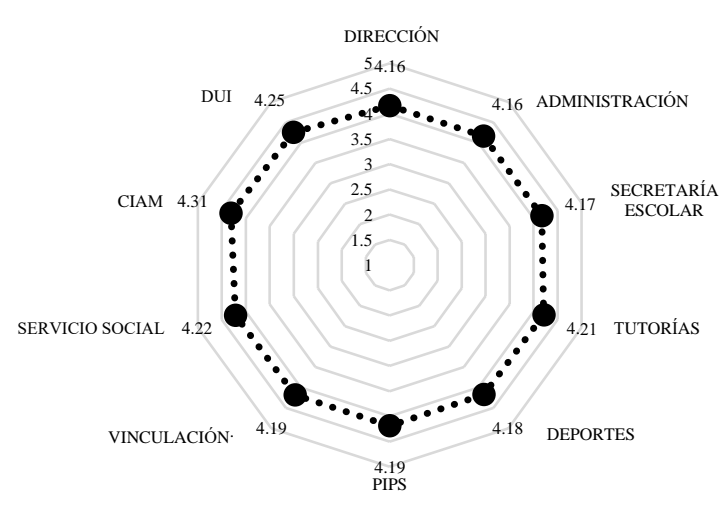

Figura 6 Dimensión de Empatía

Fuente: Elaboración propia a partir de los datos

\section{Evaluación realizada por el personal docente y administrativo}

En el caso de la evaluación realizada por los docentes y administrativos, la Secretaría Académica y la Secretaría Escolar son las áreas que tienen una calificación $(71 \%$ para ambas áreas) menor respecto a las áreas restantes, sin embargo, es importante resaltar que la calificación más alta es de un $76 \%$ obtenido por la Secretaría General. Esta información se puede observar en la Figura 7.

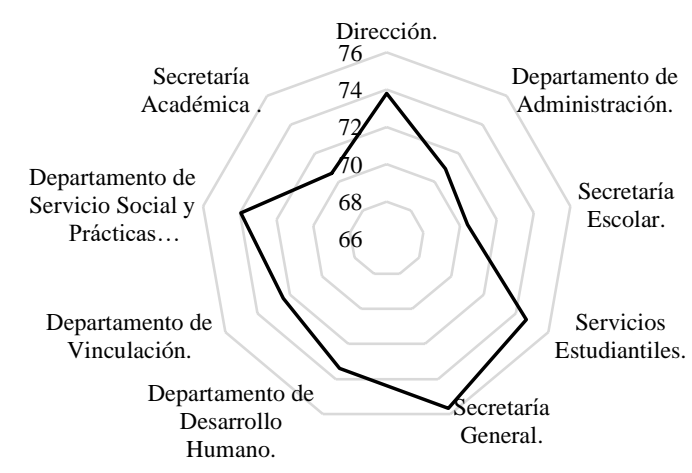

Figura 7 Promedio de áreas evaluadas

Fuente: Elaboración propia a partir de los datos.

La figura 8 muestra la evaluación obtenida de las cinco dimensiones por cada una de las áreas transversales por parte de los académicos y administrativos. Se puede observar que la evaluación de las cinco dimensiones es muy similar para todas las áreas 


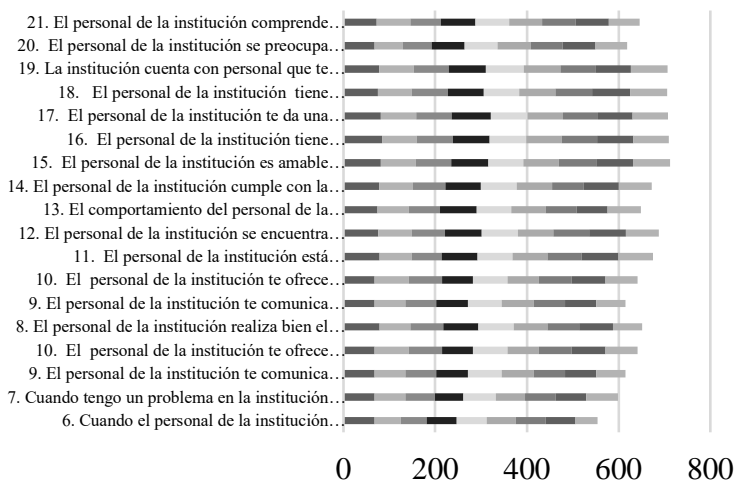

- Dirección.

- Departamento de Administración.

- Secretaría Escolar.

— Servicios Estudiantiles.

Secretaría General.

$\square$ Departamento de Desarrollo Humano.

- Departamento de Vinculación.

- Departamento de Servicio Social y Prácticas Profesionales.

Secretaría Académica .

Figura 8 Evaluación de las 5 dimensiones del modelo Servqual

Fuente: Elaboración propia a partir de los datos

\section{Conclusiones}

El modelo SERVQUAL permite evaluar la calidad en el servicio en las diferentes áreas transversales de la Institución, se puede apreciar que de las cinco dimensiones que conforman el modelo, la dimensión mejor evaluada es la de Capacidad de Respuesta; es decir, las diferentes áreas tienen la disposición para ayudar a los estudiantes de la institución. Cabe resaltar que las cuatro dimensiones restantes también se encuentran con una calificación superior a la media, por lo tanto, se concluye que los estudiantes se encuentran satisfechos con la calidad en el servicio recibido. En el análisis de la dimensión de elementos tangibles, los estudiantes se encuentran satisfechos con las instalaciones e infraestructura y el trato que recibe por parte del personal de apoyo en las diferentes áreas de la institución, sin embargo, la tecnología representa un área de oportunidad para dicha institución, esto se debe a que algunas de las carreras no cuentan con el $100 \%$ de la tecnología dentro de sus laboratorios o aulas.
Continuando con el análisis de las dimensiones restantes, cabe mencionar que las áreas que destacan en la Confiabilidad, la Capacidad de Respuesta, la Seguridad y la Empatía son las áreas del CIAM el DUI y el Servicio Social, las cuales obtuvieron mejores evaluaciones por los estudiantes sobre la calidad que brindan en el servicio respecto a las áreas restantes. Tal pareciera que las áreas de dirección y administración son evaluadas con una puntuación menor, sin embargo, las diez áreas evaluadas se encuentran por encima de la media, es decir, en promedio tienen una calificación superior a 4 puntos, lo cual indica que la percepción que tienen los estudiantes de la calidad en el servicio que ofrece la institución es buena y por lo tanto se encuentran satisfechos con el servicio brindado.

Finalmente se puede concluir que el personal docente y administrativo de la institución son mas duros al evaluar la calidad en el servicio en las áreas transversales respecto a los alumnos, por lo tanto, la percepción que tiene el personal que labora en la Institución sobre la calidad en el servicio es menor a la que tienen los alumnos de dicha institución.

\section{Referencias}

ANUIES, Asociación Nacional de Universidades e Instituciones de Educación Superior (s/f). La Educación Superior en México. Resultados y relevancia para el mercado laboral. Revista de la Educación Superior ANUIES, (29). Recuperado de http://publicaciones.anuies.mx/acervo/revsup /res029/txt1c.htm. Fecha de consulta: 15 de diciembre de 2020.

Duque O., E. J. y Diosa G., Y. (2014) Evolución conceptual de los modelos de medición de la percepción de calidad del servicio: una mirada desde la educación superior. Suma de Negocios, $5(12)$, p. 180-191. DOI: 10.1016/S2215910X(14)70040-0

Duque y Chaparro. (2012). Medición de la percepción de la calidad del servicio de educación por parte de los estudiantes de la UPTC Duitama.

Hill, C. W., Jones, L. y Gareth, R. (1996). Administración Estratégica, Un Enfoque Integrado. Santa Fé De Bogotá: Mc Graw Hill. Malcolm Peel. "Servicio al cliente". (2010) 
Nishizawa, R. M. (26 de Julio de 2018). Desarrollo del Modelo Servqual para la medición de la calidad del servicio en la empresa de publicidad Ayuda Experto. Obtenido de scielo: http://www.scielo.org.bo/scielo.php?script=sci arttext\&pid=S1994-37332014000200005

OECD, Organization for Economic Cooperation and Development (2019). Educación Superior en México. Resultados y relevancia para el mercado laboral. OECD Publishing. Disponible

en: https://www.oecd.org/centrodemexico/medi os/educacion_superior_en_mexico.pdf. Fecha de consulta: 15 de diciembre de 2020 .

Parasuraman. (1985). Differetial perceptions of suppliers and clients of industrial services.

Schiffman, L., y Kanuk, L. (2005). Comportamiento del consumidor. México: Prentice Hall.

Zeithaml, Valerie; Bitner, Mary Jo; Gremler. (2009). Marketing de servicios. México: Mc Graw Hill. Quinta edición

Zeithaml, V., Berry Ly y Parasuraman, A. (1993). "The Nature and Determinants of Customer Expectations of Service," Academy of Marketing Science, 1993, 1-12. 